ARTIGO

\title{
O programa Ciência sem Fronteiras e a falha sistêmica no ciclo de políticas públicas*
}

Cintia Denise Granja a Ana Maria Carneiro ${ }^{b}$

\section{Resumo}

Este artigo analisa o programa brasileiro de mobilidade estudantil e acadêmica Ciência sem Fronteiras, utilizando o ciclo de políticas públicas como instrumento metodológico. $\mathrm{O}$ estudo baseia-se na literatura, em documentos disponíveis sobre o programa e em um estudo de caso realizado em uma universidade brasileira. O programa mostrou fragilidades de forma sistêmica, ou seja, em todas as fases de seu ciclo, com problemas desde a identificação do problema e a formação da sua agenda, mas tangenciando também sua formulação, implementação e avaliação. Viu-se que a política foi criada e implementada de forma abrupta e que seu desenho não foi adequado para o cumprimento de seus objetivos, além de custar mais do que o planejado e não vir acompanhado de uma avaliação que contemplasse seu ciclo completo.

Palavras-chave: Ensino Superior. Avaliação. Políticas Públicas. Ciência, Tecnologia e Inovação. Mobilidade Estudantil.

\section{Introdução}

Apesar de não ser um fenômeno recente, nas últimas décadas, a mobilidade estudantil vem ganhando popularidade no setor educacional (GURUZ, 2008;

\footnotetext{
* O artigo recebeu o apoio da Fundação de Amparo à Pesquisa do Estado de São Paulo (Fapesp), sob o processo n²016/10037-7.

a United Nations University, Maastricht, Países Baixos.

b Universidade Estadual de Campinas, Campinas, SP, Brasil.
}

Recebido em: 18 set. 2018

Aceito em: 04 nov. 2019 
KNIGHT, 2005) e os incentivos dos governos para programas de intercâmbio estudantil vêm se ampliando (ENGBERG et al., 2014) ${ }^{1}$.

No Brasil, entre 2000 e 2011, houve um aumento de $71 \%$ do número de estudantes do Ensino Superior no exterior, passando de 17 mil para 29,7 mil (UIS, 2016). Apesar de tal aumento, a mobilidade estudantil no Brasil crescia a passos lentos, quando comparada a outros países, considerando sua população e a quantidade de alunos matriculados no Ensino Superior (UIS, 2016).

Foi nesse contexto que o governo brasileiro criou, em 2011, o programa de mobilidade Ciência sem Fronteiras (CsF). Congelado em 2015 e cancelado em 2017, o programa concedeu mais de 90 mil bolsas de estudo e pesquisa no Brasil e no exterior (BRASIL, 2016), aumentando significativamente a quantidade de pesquisadores e estudantes brasileiros em mobilidade e o volume de bolsas fornecidas pela Coordenação de Aperfeiçoamento de Pessoal de Nível Superior Capes (BRASIL, 2017a) e pelo Conselho Nacional de Desenvolvimento Científico e Tecnológico - CNPq (BRASIL, 2017b).

Este artigo analisa o CsF, utilizando como instrumento metodológico o conceito de ciclo de políticas públicas. Escolheu-se tal tema após analisarmos os efeitos institucionais do programa na Universidade Estadual de Campinas (GRANJA, 2018), uma das universidades brasileiras com mais beneficiários do programa: 2.384 bolsas (BRASIL, 2016). Tal pesquisa mostrou que muitos dos problemas do CsF vieram da forma como o programa foi criado e implementado, levando à hipótese de que o programa apresentou fragilidades em várias etapas de seu ciclo político.

Adicionalmente, escolheu-se esta abordagem porque, com o encerramento do CsF, torna-se possível estudar todo o seu ciclo e analisá-lo como uma política pública brasileira, de forma a analisar sua coerência e os instrumentos utilizados para alcançar seus objetivos. Trata-se de uma abordagem inovadora, visto que não foram encontradas avaliações do programa que, simultaneamente, analisassem todo o ciclo do programa e utilizassem o mesmo instrumento metodológico.

O artigo tem um caráter qualitativo e utiliza, como base, as informações disponibilizadas pelo programa (estatísticas oficiais, decretos e editais), publicações

Entende-se a mobilidade estudantil como parte das estratégias de internacionalização do Ensino Superior, que ocorre quando os indivíduos saem de seu país de origem com o propósito de estudar (VERBIK; LASANOWSKI, 2007). 
acadêmicas, relatórios e notícias ${ }^{2}$. Adicionalmente, o artigo utiliza informações provenientes do estudo de caso realizado na Universidade Estadual de Campinas (Unicamp), mencionado anteriormente ${ }^{3}$.

O artigo é composto por três seções. A primeira apresenta o conceito de ciclo de políticas públicas e descreve as suas fases. Já a segunda analisa o programa tomando como base as fases do ciclo de políticas públicas. Por fim, a última seção apresenta um balanço das informações apresentadas no decorrer do artigo.

\section{Políticas públicas e seus ciclos}

As políticas públicas são um campo de conhecimento que pretende, simultaneamente, colocar o governo em ação e/ou realizar uma análise dessa ação e, se preciso, realizar uma proposta de mudanças (SOUZA, 2006). São as ações, metas e planos que os governos traçam para alcançar o bem-estar da sociedade e o interesse público (LOPES; AMARAL; CALDAS, 2008), traduzindo "seus propósitos e plataformas eleitorais em programas e ações que produzirão resultados ou mudanças no mundo real" (SOUZA, 2006, p. 26).

De acordo com Bekkers, Fenger e Scholten (2017), o processo de decisão política foca tanto na formulação de políticas para atingir determinados objetivos, quanto na seleção de meios e instrumentos, na formulação de medidas e na utilização de recursos. Para os autores, programas políticos não se sustentam sozinhos, mas geram diferentes processos, que podem ser visualizados por meio do modelo do ciclo de políticas.

O ciclo de políticas pode ser entendido como um "esquema de visualização e interpretação que organiza a vida de uma política pública em fases sequenciais e interdependentes" (SECCHI, 2012, p. 33). A denominação das fases varia amplamente na literatura, seja em sete etapas (SECCHI, 2012), cinco (LOPES; AMARAL; CALDAS, 2008) ou quatro (VIANA, 1996). Apesar da ausência de

\footnotetext{
O artigo está fundamentado nos resultados da dissertação de mestrado da primeira autora. Para obter as informações acadêmicas, partiu-se de uma revisão sistemática em duas bases, a Biblioteca Digital Brasileira de Teses e Dissertações e o Google Scholar, utilizando os seguintes termos: "Ciência sem Fronteiras", "CsF", "Science without Borders" e "SwB". Esses dados foram complementados por informações oficiais, como decretos, editais e relatórios publicados no decorrer do programa, em grande parte disponíveis em seu website oficial e no Painel de Controle do CsF.

3 O estudo de caso foi conduzido através de entrevistas semiestruturadas com 12 coordenadores de cursos de Graduação, seis gestores de internacionalização na universidade, 10 professores e pesquisadores beneficiários de bolsas de pós-doutorado do programa e 14 coordenadores técnicos de auxílios, que levaram pesquisadores do exterior para a Unicamp. O estudo foi complementado com dados secundários (administrativos), coletados em fontes internas da Unicamp (Vice-Reitoria de Relações Internacionais, Comissão Permanente para os Vestibulares e Diretoria Acadêmica) e em fontes externas (dados das agências de fomento e das plataformas Bolsistas pelo Mundo e Painel de Controle do CsF).
} 
consenso, todos os autores apontam as fases de formulação, implementação e avaliação (FREY, 2000). As demais fases variam entre identificação do problema, formação/construção da agenda, formulação da política/de alternativas, tomada de decisão e extinção da política. Segundo Cairney (2012), o importante dessa literatura é entender que o estudo de uma política não acaba quando um problema é levantado e uma decisão é tomada, mas, ao contrário, esse é apenas o início do processo político.

Apesar desse modelo ser alvo de diversas críticas, principalmente no que tange à sua simplicidade, ele é um instrumento bastante didático para a análise da vida de uma política (ARAÚJO; RODRIGUES, 2017; FREY, 2000). Ao tentar mostrar como os governos tentam transformar demandas em políticas públicas, o modelo apresentase de grande utilidade como ponto de partida para organizar o estudo do processo de elaboração de políticas, sendo útil também para comparar sistematicamente diferentes políticas (ARAÚJO; RODRIGUES, 2017; CAIRNEY, 2012).

O primeiro elemento do ciclo de políticas é a identificação de um problema e a construção da agenda política. Quando um problema é identificado por um ator político que tenha interesse na sua resolução, tal ator pode tentar inseri-lo na lista de prioridades, ou seja, na agenda política/governamental (LOPES; AMARAL; CALDAS, 2008; SECCHI, 2012; VIANA, 1996). Uma questão torna-se prioritária quando existe "vontade política, mobilização popular e percepção de que os custos de não resolver o problema serão maiores do que os custos de resolvê-lo" (LOPES; AMARAL; CALDAS, 2008, p. 12). Os problemas identificados entram e saem das agendas, ganhando notoriedade e relevância, e depois desinflando (SECCHI, 2012).

Conforme Lopes, Amaral e Caldas (2008, p. 12), "a partir do momento em que uma situação é vista como problema [...] é necessário definir as linhas de ação que serão adotadas para solucioná-los". A segunda etapa é, portanto, a formulação da política, que consiste em formular alternativas e escolher entre elas (tomada de decisão) (VIANA, 1996). É nessa etapa em que se elaboram os métodos, programas, estratégias ou ações para cumprir os objetivos pretendidos e que se avaliam os custos e as consequências das alternativas propostas pelos atores envolvidos (SECCHI, 2012).

Para Lopes, Amaral e Caldas (2008), um bom processo de elaboração de uma política compreende as seguintes etapas: i) conversão de estatísticas em informação relevante para o problema; ii) análise das preferências dos autores; e iii) ação baseada no conhecimento adquirido. Para eles, para que se facilite a elaboração 
de propostas, o responsável pela elaboração da política deve procurar envolver os atores que serão responsáveis pela sua implementação.

A terceira etapa é a implementação da política, que ocorre após a tomada de decisão, sendo a etapa em que se transforma o planejamento e a escolha em atos (LOPES; AMARAL; CALDAS, 2008). Existem dois tipos de modelo de implementação de políticas: i) o modelo top-down, quando a política é implementada no sentido "do governo para a população", havendo uma separação clara entre a tomada de decisão e a implementação; e ii) o modelo bottom-up, que ocorre "da população para o governo", de forma descentralizada, dando maior liberdade para que os atores se auto-organizem e modelem a implementação da política (LOPES; AMARAL; CALDAS, 2008; SECCHI, 2012).

Conforme Cairney (2012), uma falha de implementação poderia ocorrer devido a três grandes fatores: má execução (a política não é realizada como o proposto), má política (a política é bem executada, mas falha ao atingir os resultados desejados) ou má sorte (a política é minada por fatores externos aos seus formuladores).

A quarta etapa é a avaliação, que é a fase "em que o processo de implementação e o desempenho da política são examinados com o intuito de conhecer melhor o estado da política e o nível de redução do problema que a gerou" (SECCHI, 2012, p. 49). Ela pode ser feita antes (ex-ante), durante (in itinere) ou depois (ex-post) da implementação, podendo utilizar diferentes critérios e gerar resultados que podem levar à continuação, à reestruturação ou mesmo à extinção da política (FREY, 2000; JANNUZZI, 2011; SECCHI, 2012).

Apesar de a fase de avaliação ser "imprescindível para o desenvolvimento e para a adaptação contínua das formas e instrumentos da ação pública" (FREY, 2000, p. 229), realizar avaliações completas e significativas é uma tarefa difícil, dados seus altos custos e dificuldades decorrentes da falta de clareza dos objetivos da política, da multicausalidade, da possível resistência dos indivíduos que serão avaliados, do tempo de maturação da política ou mesmo da dificuldade em se encontrar dados sistematizados sobre as políticas implementadas (JANNUZZI, 2011; SECCHI, 2012).

\section{O programa Ciência sem Fronteiras e o Ciclo de Políticas}

Esta seção discute o programa $\mathrm{CsF}$ utilizando o instrumental do ciclo de políticas. Apresentam-se informações sobre as fases de identificação do problema e construção da agenda política, formulação, implementação e avaliação do programa. 


\subsection{Identificação do problema e construção da agenda política}

O programa CsF foi um programa de mobilidade estudantil e acadêmica que tinha a meta de conceder 101 mil bolsas no exterior e no país, meta que possivelmente fazia referência à iniciativa americana 100,000 Strong in the Americas (KNOBEL, 2012) ${ }^{4}$.

O CsF foi lançado pelo governo federal em julho de 2011 (BRASIL, 2011a) e instituído poucos meses depois (BRASIL, 2011b). Posteriormente à sua criação, o programa foi enunciado como parte da estratégia de fortalecer a base de sustentação da política de Ciência, Tecnologia e Inovação brasileira, dado o déficit de engenheiros formados no país e o baixo número de bolsas de estudo no exterior concedidas pelas agências de fomento federais brasileiras (BRASIL, 2012a).

Ressalta-se que a inserção do programa na agenda política brasileira da época foi decorrente da soma de dois fatores: o cenário brasileiro de forte otimismo em relação à recursos orçamentários, haja vista a expectativa de abundância de recursos decorrentes da descoberta da camada de Pré-Sal no Brasil e a grande vontade política presidencial, que colocou o programa como questão prioritária diretamente na Casa Civil (CRUZ, 2016).

\subsection{Formulação e Implementação do Ciência sem Fronteiras}

O programa foi planejado para conceder bolsas de estudo em sete modalidades, cinco em "instituições de excelência no exterior" (Graduação Sanduíche, Educação Profissional e Tecnológica, Mestrado Profissional, Doutorado Sanduíche e Doutorado Pleno) e duas no país (Pesquisador Visitante Especial e Jovens Talentos).

Ele foi colocado no Brasil sob a responsabilidade da Capes e do CNPq que, com seu anúncio repentino, tiveram que se esforçar para implementar o programa às pressas (CRUZ, 2016; KNOBEL, 2012; VELHO; RAMOS, 2014). O programa baseou-se também em agências parceiras no exterior (agências de placement),

\footnotetext{
O programa foi anunciado alguns dias após a visita do então presidente americano Barack Obama, que desafiou a então presidente do Brasil Dilma Rousseff a corresponder às iniciativas americanas com relação à mobilidade de estudantes para América Latina (BRASIL, 2012b; KNOBEL, 2012). Vale ressaltar, entretanto, que o programa americano era baseado principalmente em parcerias público-privada (USDOS, 2013), o que não foi o caso do CsF, como se verá.
} 
que tinham como função alocar os estudantes de Graduação nas instituições estrangeiras (MANÇOS, 2017).

A gestão do programa contou com um Comitê de Acompanhamento e Assessoramento (CAA), com a atribuição de acompanhar e de avaliar o desenvolvimento do programa (BRASIL, 2011b). Apesar disso, não foram encontrados indícios de que o CAA permaneceu ativo durante todo o programa e nem relatórios realizados pelo comitế. $\mathrm{O}$ programa contou também com um Comitê Executivo (CE), que deveria estabelecer o cronograma de execução, bem como critérios de seleção de bolsistas e de instituições participantes, além de definir valores e períodos das bolsas e apoio a projetos e identificar centros e lideranças no exterior de interesse prioritário, em áreas e setores selecionados para estabelecimento de cooperação e treinamento (BRASIL, 2011b). Ao contrário do CAA, o CE realizou uma série de reuniões ao decorrer do programa, realizando tarefas, como a aprovação de modalidades e de revisão de metas ${ }^{6}$. Em relação à formulação do CsF, apesar da menção de ambos os comitês no decreto de criação do programa, não foram encontrados indícios de que tenha havido discussão com os principais stakeholders antes do anúncio do programa, bem como entre o seu anúncio e sua criação.

Assim como com o que ocorreu com os dois comitês do CsF, os estudos sobre o programa e a pesquisa de campo na Unicamp (GRANJA, 2018) também sugeriram que o programa foi instaurado pelo governo sem ter sido discutido com as instituições de Ensino Superior brasileiras, o que gerou dificuldades na fase de implementação do programa (BIDO, 2015; CUNHA, 2016; VELHO; RAMOS, 2014). Assim, o programa seguiu uma abordagem top-down, não envolvendo, em sua formulação, os principais atores relacionados à internacionalização do Ensino Superior brasileiro (instituições de Ensino Superior e agências de fomento), que foram envolvidos no programa somente na etapa seguinte. Ressalta-se que, apesar da falta de envolvimento de stakeholders ser uma abordagem comum em programas de mobilidade financiados pelo governo (GRIECO, 2015), essa prática vai de encontro com o que sugere a literatura sobre políticas públicas vista anteriormente (LOPES; AMARAL; CALDAS, 2008; SECCHI, 2012).

Além do baixo envolvimento dos atores, podemos destacar, ainda, outras fragilidades da fase de formulação do programa. O primeiro ponto é que o programa tinha uma lista extensa e ambiciosa de objetivos, o que incluía ampliar

\footnotetext{
5 Foram localizadas informações somente de duas reuniões: Brasil (2012c; 2014).

6 Indícios sobre a atuação do CE podem ser encontrados em: Brasil [201-a;201-b].
} 
a mobilidade estudantil e acadêmica e aumentar a cooperação técnico-científica internacional, além de contribuir para a internacionalização das instituições de Ensino Superior e centros de pesquisas brasileiros, propiciar maior visibilidade internacional à pesquisa brasileira, estimular as pesquisas aplicadas no país e aumentar a competitividade das empresas brasileiras (BRASIL, 2011b). No entanto, os mecanismos utilizados pelo programa não eram suficientes para abarcar todos os objetivos, especialmente ao considerar que a maior modalidade (Graduação Sanduíche) não previa atividades de pesquisa, nem no Brasil e nem no exterior. Além disso, vale ressaltar que a lista de objetivos do programa foi instituída alguns meses após o anúncio do CsF, assim como a definição de áreas prioritárias contempladas pelo programa foi feita um ano após o seu início (BRASIL, 2013).

Ainda com relação à formulação do programa, foram encontrados dois erros estratégicos na definição das instituições de destino. Em primeiro lugar, as instituições de Ensino Superior brasileiras não puderam decidir para onde enviar seus estudantes, o que, segundo Grieco (2015), poderia fazer com que as agências governamentais tivessem mais dificuldades ao escolher os destinos de estudo, por falta de conhecimento de como os diferentes programas de estudo dessas instituições se encaixariam nos interesses e no campo de estudo dos bolsistas.

Além disso, o processo não levou em conta os mecanismos de admissão das universidades no exterior, o que já havia sido previsto por Castro et al. (2012), que argumentaram que garantir aceitação dos alunos em universidades de primeira linha seria uma tarefa difícil, já que essas instituições não costumam delegar a seleção de seus candidatos a agências de placement. Esse problema fica claro ao observar as instituições de destino disponíveis nos editais (ENGBERG et al., 2014) e com o fato de que poucos bolsistas foram enviados para universidades bem posicionadas em rankings universitários, o que era uma das metas do programa (CCT, 2015).

Outro problema na formulação que gerou dificuldades na implementação posterior do programa foi a ausência de diagnóstico sobre o baixo nível de fluência na língua estrangeira dos potenciais beneficiários do programa, levando à falta de planejamento de ações prévias à mobilidade que pudessem minimizar tais dificuldades (como estratégias de internacionalização "em casa"), o que já havia sido levantado por pesquisadores desde o início do programa (CASTRO et al.,

\footnotetext{
Estratégias de internacionalização 'em casa' são as atividades desenvolvidas dentro dos campi das instituições, servindo de suporte à mobilidade estudantil, podendo ser focadas em melhoria de currículo, no entendimento global ou no desenvolvimento de habilidades interculturais (BEELEN; JONES, 2015; DE WIT et al., 2015; KNIGHT, 2004).
} 
2012). Esse problema tornou-se visível de três formas. A primeira é que Portugal foi um dos principais destinos, mesmo com apenas dois editais abertos, ambos em 2012. A segunda é que, conforme Marques (2017), a falta de proficiência dos estudantes fez com que o CsF despendesse R $\$ 976$ milhões com cursos de idiomas ministrados antes e durante a vigência das bolsas. Por fim, apesar desse esforço, diversas avaliações do programa nas instituições de Ensino Superior mencionaram que houve dificuldades de aprendizado e aproveitamento da experiência no exterior decorrentes de deficiências no idioma estrangeiro (ATHAYDE, 2016; BORGES, 2015; FAGUNDES; LUCE; SILVEIRA, 2019; LAGE, 2015; TONELLI; WINGLER; UEBE MANSUR, 2016).

Adicionalmente, ressalta-se a fragilidade em termos de estimativa dos custos do programa e de onde viriam os recursos para viabilizá-lo. Conforme seu decreto de criação, o programa deveria ser custeado "por dotações orçamentárias da União consignadas anualmente aos órgãos e entidades envolvidos no Programa, [...] e por outras fontes de recursos, provenientes de entidades públicas e privadas" (BRASIL, 2011 b, p. 7). No entanto, o programa, que tinha um custo inicial estimado em 3 bilhões de reais (BRASIL, 2011c), custou muito mais e utilizou recursos de forma indevida, como será visto, com mais detalhes, a seguir. Além disso, adiciona-se que não foram encontrados indícios de que houve alguma negociação das taxas acadêmicas com as universidades de destino, que receberam do programa um total de 5,8 bilhões de reais entre 2012 e 2016 (MARQUES, 2017).

Para selecionar os beneficiários do programa, foram utilizados diferentes critérios, a depender da modalidade. No caso de estudantes de Graduação, segundo o manual de candidatura do CsF (BRASIL, 2015), o estudante deveria se inscrever no processo seletivo interno de sua instituição de origem e, posteriormente, se inscrever no website do programa. Cabia, então, à instituição de origem, homologar a candidatura do aluno selecionado no processo interno. A seguir, os candidatos seriam avaliados pelas agências de fomento e os aprovados seguiriam para alocação nas instituições de destino, feita através das agências parceiras em cada país. O aceite final seria dado pela instituição de destino. A escolha de onde os estudantes de Graduação Sanduíche iriam - uma das partes mais estratégicas do programa - ficou fora das decisões dos atores mais relevantes, ou seja, da Capes, do CNPq e das instituições de origem dos beneficiários.

Já para a Pós-Graduação, as instituições de destino seriam analisadas por comissões de especialistas da Capes e do CNPq, com base nas propostas apresentadas pelos candidatos (CCT, 2015). Para o Mestrado Profissional, a seleção era feita por meio da Capes e de uma agência do país de destino. No caso de bolsas de 
Doutorado Pleno, Doutorado Sanduíche e Pós-Doutorado no Exterior, os candidatos precisariam de admissão prévia na instituição de destino para a solicitação da bolsa pelo programa. Para as bolsas Jovens Talentos e Pesquisador Visitante Especial, as propostas deveriam ser encaminhadas por um pesquisador brasileiro, que acompanharia a pesquisa no país.

De acordo com os dados disponibilizados pelo governo federal no Painel de Controle do Ciência sem Fronteiras (BRASIL, 2016), até janeiro de 2016, foram implementadas 92.880 bolsas pelo CNPq e pela Capes, sendo $79 \%$ dessas para Graduação Sanduíche no Exterior, 10\% para Doutorado Sanduíche no Exterior e o restante, para as demais modalidades. Com exceção das bolsas de Graduação Sanduíche (que ultrapassaram em 15\% a meta inicial de 64 mil bolsas), todas as modalidades ficaram abaixo da meta inicial do programa, especialmente as modalidades de atração de cientistas que, em janeiro de 2016, tinham atingido respectivamente apenas $25 \%$ e $39 \%$ da meta inicial.

O custo médio por bolsista ficou entre 45 mil e 321 mil reais, valor a depender da modalidade (CCT, 2015). O programa custou, até maio de 2017, 13,2 bilhões de reais, valor que, ao término do pagamento das bolsas vigentes em 2020, deve aproximar-se de 20 bilhões de reais (quase sete vezes mais do que o estimado) (MARQUES, 2017). Do total gasto com o programa, $48 \%$ destinaram-se às bolsas, $44 \%$ aos pagamentos de taxas acadêmicas às universidades estrangeiras e 7\% aos cursos de idiomas (MARQUES, 2017). Em 2015, mais de 60\% dos recursos do programa vinham do Ministério da Educação (MEC) e mais de 30\% do Ministério da Ciência, Tecnologia e Inovação (MCTI) (CCT, 2015). O custo total do programa correspondeu a mais de 15 vezes o orçamento empenhado do CNPq, em 2016, e a 50\% do orçamento da Capes em 2015 (MARQUES, 2017). Além disso, apesar de o programa ter como meta que a iniciativa privada custeasse $25 \%$ das bolsas, até outubro de 2015 , somente $16,7 \%$ das bolsas vieram de recursos privados (CCT, 2015).

Com relação ao financiamento, uma crítica presente na literatura, realizada também pelo próprio Tribunal de Contas da União, foi a utilização dos recursos do Fundo Nacional de Desenvolvimento Científico e Tecnológico (FNDCT) para o custeio das bolsas, visto que o uso desses recursos foi encarado como indevido, já que as bolsas de Graduação Sanduíche (maior modalidade e gasto do programa) não representariam esforço em pesquisa e desenvolvimento, mas atividades de formação (KOELLER; VIOTTI; RAUEN, 2016). Em 2014, mais

${ }^{8}$ Cálculos realizados com base em CCT (2015) e CsF (2016). 
de um terço dos recursos do FNDCT foi alocado para o programa, montante que cresceu para mais de 40\% em 2015 (R\$ 367,1 milhões) (CCT, 2016).

\subsection{Avaliações do Ciência sem Fronteiras}

No que tange à avaliação do programa por parte de sua gestão, não foi possível localizar um relatório público de avaliação do programa realizado pelo CAA, que tinha como uma de suas funções acompanhar e avaliar o desenvolvimento do programa, como visto anteriormente.

Até o segundo semestre de 2018, houve somente a divulgação de um relatório realizado pela Comissão de Ciência, Tecnologia, Inovação, Comunicação e Informática do Senado Federal (CCT, 2015), que foi publicado antes do término do programa. O programa também foi citado em dois julgados do Tribunal de Contas da União (TCU), o Acórdão ${ }^{\circ}$ 1.232/2014, que mencionou a necessidade de realizar-se uma auditoria específica para o programa e o Acórdão n ${ }^{\circ}$ 500/2015, que determinou providências para realização de fiscalização sobre a estrutura gerencial do programa pela Controladoria Geral da União (CGU) (CCT, 2015). No entanto, apesar da determinação do TCU, também não foi localizado relatório de fiscalização específico para o CsF.

O relatório da CCT (2015) focou em apresentar dados gerais sobre seu funcionamento, implementação e execução orçamentária. A partir de todas as informações analisadas, tal relatório sugeriu que se desse continuidade ao $\mathrm{CsF}$, mesmo em um cenário de crise nacional. O relatório também fez outras recomendações, como: transformar o $\mathrm{CsF}$ em uma política de Estado; intensificar a busca de parceiros do setor privado; priorizar a Pós-Graduação; aproximar as universidades brasileiras e as estrangeiras; introduzir critérios de equidade na concessão de bolsas; criar bolsas parciais ou mobilidade via empréstimos; ampliar o incentivo e reduzir os entraves burocráticos para atração de cientistas; ampliar bolsistas em instituições mais bem avaliadas nos rankings internacionais; oferecer maior suporte aos bolsistas; investigar o não aproveitamento de créditos de disciplinas nas instituições brasileiras; criar mecanismos para avaliação do programa e incentivar as universidades para avaliarem o programa; e ampliar os investimentos em laboratórios de instituições nacionais. Ao se observar a lista de sugestões, é possível perceber que as sugestões vão no sentido de atacar os problemas ocorridos no programa.

Apesar da escassez de avaliações oficiais, há uma extensa literatura sobre o CsF. A maior parte dos estudos concentrou-se na análise dos benefícios individuais mostrando resultados positivos em termos de ampliação da visão de mundo e 
de enriquecimento intercultural (BIDO, 2015; LAGE, 2015), desenvolvimento humano (CICHOSKI, 2017), aumento da autonomia (CHAVES, 2015), ampliação das competências linguísticas (BIDO, 2015; CICHOSKI, 2017), amadurecimento acadêmico (CHAVES, 2015; MILHOMEM, 2016) e efeitos positivos na formação profissional dos beneficiários (PEREIRA, 2013; RAMOS, 2016)

Já com relação às instituições de origem, notou-se que os efeitos do programa foram limitados, pois houve pouco aproveitamento das experiências dos beneficiários após seu retorno às universidades (BIDO, 2015; LIMA, 2016; RESCHK; BIDO, 2017). O estudo na Unicamp mostrou que os efeitos de curto-prazo para a universidade foram tímidos, ocorrendo em termos da sistematização dos pedidos de intercâmbio e da padronização dos critérios de elegibilidade, que continuaram a servir como parâmetros para outros programas de mobilidade após o término do CsF. Alguns estudos sobre o CsF nas instituições de ensino apontaram efeitos positivos do programa, como a melhoria da preparação e dinâmica das equipes de relações internacionais das universidades (BIDO, 2015; RESCHK; BIDO, 2017), a abertura de canais de comunicação entre universidades brasileiras e as estrangeiras (RESCHK; BIDO, 2017; STALLIVIERI, 2015), além da possibilidade de desenvolvimento de projetos internacionalizados de cooperação em pesquisa (SAENGER; TEIXEIRA, 2018). Apesar disso, efeitos negativos não esperados foram encontrados na Unicamp, como o recuo de algumas parcerias pré-existentes e a redução das inscrições dos alunos em outros programas de mobilidade (em que as taxas acadêmicas eram inexistentes ou mais baixas), durante o período do $\mathrm{CsF}$ (GRANJA, 2018).

Em termos de análise sobre os impactos do CsF para o país, há uma escassez de estudos. Fernández (2016) sugere que o programa pode ter elevado a capacidade científica nacional devido ao investimento na formação de pessoal no exterior. Outros estudos mencionaram também uma possível melhoria da visibilidade da Educação Superior brasileira e o fortalecimento ou a inserção de instituições brasileiras em programas de cooperação no campo de pesquisa (MANÇOS, 2017; SAENGER, 2016). No entanto, esta é uma frente de pesquisa que merece mais estudos, dado o pouco tempo desde o término do CsF, além de não ter havido um esforço nacional para se avaliar resultado e impacto após sua descontinuação.

\section{Considerações finais}

A partir da análise do programa CsF, este artigo conclui que o programa apresentou fragilidades de forma sistêmica, ou seja, em todas as fases de seu ciclo, com as 
dificuldades iniciando na fase de identificação de um problema, mas também passando pela formulação, pela implementação e pela avaliação da política.

Com relação à identificação de um problema e a construção da agenda política, viu-se que o programa foi incluído na agenda repentinamente (VELHO; RAMOS, 2014), através de uma abordagem top-down, sem a identificação clara de um problema que devesse ser solucionado com a criação do programa. Pode-se dizer que sua criação foi de encontro com a literatura sobre o ciclo de políticas apresentada anteriormente, que sugere que uma política leve em conta o uso de dados anteriores e a preferência dos atores envolvidos (LOPES; AMARAL; CALDAS, 2008). Assim, não só não houve alinhamento com os atores responsáveis pela sua implementação, ou justificativa que embasasse a meta de bolsas, como também a elaboração do programa não levou em conta que as bolsas sanduíche modalidade mais utilizada no programa - não haviam sido propriamente avaliadas até então (VELHO; RAMOS, 2014).

Em termos da formulação do CsF, notou-se uma inconsistência no modo como o programa foi desenhado em relação aos objetivos estabelecidos. Os dados da distribuição de bolsas (BRASIL, 2016) mostram que o desenho do programa não tinha instrumentos para abarcar sua extensa lista de objetivos. Acredita-se, assim, que os objetivos foram elaborados mais com o intuito de justificar o programa, após a sua criação, do que para auxiliar na sua estruturação.

Foram encontradas algumas falhas durante a implementação do programa, em grande parte, decorrentes de fragilidades ocorridas durante os estágios anteriores da política. Houve precariedade no seu planejamento e ausência de uma avaliação ex-ante, o que poderia ter evitado o problema da baixa proficiência de idioma dos estudantes brasileiros e a dificuldade de admitir estudantes em universidades de excelência no exterior. Além de não atingir a meta de financiamento privado, teve um custo muito acima do esperado e utilizou recursos públicos de forma indevida. Não foram analisadas alternativas que pudessem ter um melhor custo-benefício como, por exemplo, negociar as taxas das universidades no exterior - o que era prática recorrente tanto das agências de fomento, quanto das universidades -, buscar por fontes alternativas de financiamento ou realizar a internacionalização 'em casa' antes da mobilidade dos beneficiários.

Assim, o desenvolvimento e a implementação do CsF vão de encontro com a literatura sobre políticas públicas, que argumenta que o sucesso de um programa está diretamente relacionado à capacidade do governo de mobilizar recursos de 
modo que os objetivos determinados e as alternativas escolhidas possam ser devidamente atingidos (BEKKERS; FENGER; SCHOLTEN, 2017).

Em termos de avaliação, viu-se que, apesar de seus altos custos, o programa não foi acompanhado de avaliações ex-ante ou ex-post. Como em outras políticas públicas brasileiras, essa etapa acabou sendo deixada à margem, o que compromete a prestação de contas perante a sociedade (CCT, 2015). Acredita-se, adicionalmente, que a forma abrupta como o programa foi criado também dificultou o planejamento de uma avaliação que contemplasse todo o seu ciclo. É importante mencionar que seu surgimento, sua implementação e sua finalização ainda são recentes e que o período, desde seu término, foi marcado por grande instabilidade política no país.

Por fim, ressalta-se que os maiores beneficiados do programa foram os receptores das bolsas, que desfrutaram de privilégios em todas as dimensões. As instituições de Ensino Superior estrangeiras também usufruíram grandemente dos recursos do programa, recebendo uma grande parcela de recursos públicos brasileiros. Já para as instituições de Ensino Superior brasileiras os resultados foram limitados, sendo importante mencionar que o programa não previa mecanismos para que ele pudesse ter um impacto maior e mais duradouro nessas instituições, como o fomento de cooperação em pesquisa. Já os retornos do ponto de vista nacional ainda precisam ser avaliados no médio-longo prazo.

Apesar de todas as fragilidades, não se pode deixar de reconhecer a relevância do programa que, como visto, foi a maior política pública brasileira de envio de pessoas para o exterior nas últimas décadas. A crítica que se coloca, por fim, é que um programa dessa magnitude deveria ter sido planejado de forma mais sistemática, sendo implementado de forma escalada e com um acompanhamento constante, permitindo que ele pudesse ser mais sustentável no tempo e, também, que não precisasse ser removido da agenda política. 


\title{
The Science without Borders program and the systemic failure in the public policy cycle
}

\begin{abstract}
This article analyzes the Brazilian student and academic mobility program called Science without Borders, using the public policy cycle methodological tool. The study is based on the program's available literature and documents and on a case study carried out in a Brazilian university. The program showed weaknesses in a systemic way, in other words, in all phases of its cycle, with problems occurring since the identification of the problem and formation of its agenda, but also in its formulation, implementation and evaluation. It was noticed that the policy was created and implemented abruptly and that its design was not adequate for the fulfillment of its objectives, also costing more than planned and not contemplating an evaluation of its complete cycle.
\end{abstract}

Keywords: Higher Education. Evaluation. Public Policy. Science, Technology and Innovation. Student Mobility.

\section{El programa Ciencia sin Fronteras y la falla sistémica en el ciclo de políticas públicas}

\section{Resumen}

Este artículo tiene como objetivo analizar el programa brasileño de movilidad estudiantil y académica Ciencia sin Fronteras, utilizando el ciclo de políticas públicas como instrumento metodológico. El estudio se basa en la literatura y documentos disponibles sobre el programa y en un estudio de caso realizado en una universidad brasileña. El programa mostró debilidades de manera sistémica, es decir, en todas las fases de su ciclo, con problemas desde la identificación del problema y la formación de su agenda, pero también tangente a su formulación, implementación y evaluación. Se descubrió que la política se creó e implementó abruptamente y que su diseño no era adecuado para cumplir con sus objetivos, costando más de lo planeado y no vino acompañado de una evaluación que incluyera su ciclo completo.

Palabras clave: Enseñanza Superior. Evaluación. Políticas Públicas. Ciencia, Tecnología e Innovación. Movilidad Estudiantil. 


\section{Referências}

ARAÚJO, L.; RODRIGUES, M. L. Modelos de análise das políticas públicas. Sociologia, Problemas e Práticas, Lisboa, n. 83, p. 11-35, fev. 2017. Disponível em: https://journals.openedition.org/spp/2662. Acesso em: 29 maio 2018.

ATHAYDE, A. L. M. Uma avaliação dos impactos do programa ciência sem fronteiras na perspectiva de beneficiários das instituições federais de ensino superior de Montes Claros - MG. 2016. Trabalho de Conclusão de Curso (Pós Graduação em Administração Pública) - Universidade Federal de Viçosa, Viçosa, 2016.

BEELEN, J.; JONES, E. Redefining internationalization at home. In: CURAJ, A. et al. (eds.). The European higher education area. [S. 1.]: Springer, 2015. p. 59-72.

BEKKERS, V., FENGER, M.; SCHOLTEN, P. Public policy in action: perspectives on the policy process. Cheltenham: Edward Elgar, 2017.

BIDO, M. C. F. Ciência com fronteiras: a mobilidade acadêmica e seus impactos. 2015. Dissertação (Mestrado em Gestão Educacional) Universidade do Vale do Rio dos Sinos, São Leopoldo, RS, 2015.

BORGES, R. A. A interseccionalidade de gênero, raça e classe no Programa Ciência Sem Fronteiras: um estudo sobre estudantes brasileiros com destino aos EUA. 2015. Dissertação (Mestrado em Educação) - Faculdade de Educação, Universidade de Brasília, Brasília, DF, 2015.

BRASIL. Decreto No 7.642, de 13 de dezembro de 2011. Institui o Programa Ciência sem Fronteiras. Diário Oficial da União, 14 dez. 2011 b.

BRASIL Portaria Interministerial $N^{o} 1$, de 9 de janeiro de 2013. Ficam instituídas as áreas e temas prioritários de atuação do Programa Ciência sem Fronteiras. Diário Oficial da União, 11 jan. 2013.

BRASIL. Ciência sem Fronteiras. Desenvolvimento tecnológico. [201-b]. Disponível em: http://www.cienciasemfronteiras.gov.br/web/csf/ desenvolvimento-tecnologico. Acesso em: 23 jun. 2018.

BRASIL. Ciência sem Fronteiras. Disponibilizadas as primeiras 2 mil bolsas do Programa Ciência sem Fronteiras, Brasília, DF, 17 ago. 2011a. Disponível em: http://cienciasemfronteiras.gov.br/web/csf/noticias/-/asset_publisher/ 
Dh91/content/disponibilizadas-as-primeiras-2-mil-bolsas-do-programaciencia-sem-fronteiras?redirect $=\mathrm{http} \% 3 \mathrm{~A} \% 2 \mathrm{~F} \% 2 \mathrm{~F}$ cienciasemfronteiras.gov. br\%2Fweb\%2Fcsf\%2Fnoticias\%3Fp_p_id\%3D101_INSTAN. Acesso em: 26 set. 2017.

BRASIL. Ciência sem Fronteiras. Metas. [201-a]. Disponível em: http://www. cienciasemfronteiras.gov.br/web/csf/metas. Acesso em: 23 jun. 2018.

BRASIL. Ciência sem Fronteiras. Painel de controle do Programa Ciência sem Fronteiras, Brasília, DF, 2016. Disponível em: http://www. cienciasemfronteiras.gov.br/web/csf/painel-de-controle. Acesso em: 21 mar. 2016.

BRASIL. Ciência sem Fronteiras. País terá $R \$ 3,16$ bi para formar cientistas. Brasília, DF, 28 jul. 2011c. Disponível em: http://www. cienciasemfronteiras.gov.br/web/csf/views/-/journal_content/56_INSTANCE_ VF2v/214072/230025. Acesso em: 22 jun. 2018.

BRASIL. Ciência sem Fronteiras. Presidente do CNPq ressalta a importância do Ciência sem Fronteiras nos EUA. Brasília, DF, 5 mar.

2012b. Disponível em: http://cienciasemfronteiras.gov.br/web/csf/noticias/-/ asset_publisher/Dh91/content/presidente-do-cnpq-ressalta-a-importancia-dociencia-sem-fronteiras-nos-eua;jsessionid $=726 \mathrm{C} 91 \mathrm{~F} 73746 \mathrm{~B} 0 \mathrm{~A} 6729 \mathrm{~A} 34 \mathrm{E} 882$ 19F27F?redirect=http $\% 3 \mathrm{~A} \% 2 \mathrm{~F} \% 2 \mathrm{Fcienciasemfronteiras.gov.br} \% 2 \mathrm{~F}$. Acesso em: 4 jun. 2018.

BRASIL. Ministério da Ciência, Tecnologia e Inovação - MCTI. Estratégia Nacional de Ciência, Tecnologia e Inovação 2012 - 2015: balanço das atividades estruturantes 2011. Brasília, DF, 2012a.

BRASIL. Ministério da Ciência, Tecnologia, Inovações e Comunicações. Conselho Nacional de Desenvolvimento Científico e Tecnológico - CNPq. Dados abertos. Brasília, DF, 2017b. Disponível em: http://cnpq.br/dados_ abertos. Acesso em: 10 jan. 2017.

BRASIL. Ministério da Educação. Comitê de acompanhamento do programa faz primeira reunião. 2012c. Disponível em: http://portal.mec.gov. br/ultimas-noticias/212-educacao-superior-1690610854/17775-comite-deacompanhamento-do-programa-faz-primeira-reuniao. Acesso em: 23 jun. 2018.

BRASIL. Ministério da Educação. Coordenação de Aperfeiçoamento de Pessoal de Nível Superior - Capes. Comitê de Acompanhamento e 
Assessoramento do CsF se reúne na Capes. 2014. Disponível em: http://www.capes.gov.br/36-noticias/5869-comite-de-acompanhamento-eassessoramento-do-csf-se-reune-na-capes. Acesso em: 23 jun. 2018.

BRASIL. Ministério da Educação. Coordenação de Aperfeiçoamento de Pessoal de Nível Superior - Capes. Manual de candidatura: programa de Graduação sanduíche. Brasília, DF, 2015. Disponível em: http://www.cienciasemfronteiras.gov.br/c/document_library/ get_file?uuid=2786a5fd-93e2-4eff-a069-a2448fc5ed11\&groupId=214072. Acesso em: 25 nov. 2017.

BRASIL. Ministério da Educação. Coordenação de Aperfeiçoamento de Pessoal de Nível Superior - Capes. Geocapes. Concessão de bolsas de Pós-Graduação da Capes no Brasil. Brasília, DF, 2017a. Disponível em: http:// geocapes.capes.gov.br/geocapes2/. Acesso em: 10 jan. 2017.

CAIRNEY, P. Understanding public policy: theories and issues. Houndmills: Palgrave Macmillan, 2012.

CASTRO, C. M. et al. Cem mil bolsistas no exterior. Interesse Nacional, abr.-jun. 2012. Disponível em: http://www.schwartzman.org.br/simon/cemmil. pdf. Acesso em: 22 mar. 2016.

CHAVES, G. M. N. As bolsas de Graduação-sanduíche do Programa Ciência sem Fronteiras: uma análise de suas implicações educacionais. 2015. Dissertação (Mestrado em Educação) - Universidade Católica de Brasília, Brasília, DF, 2015.

CICHOSKI, P. S. B. Experiências de internacionalização e desenvolvimento humano na Educação Superior. 2017. Dissertação (Mestrado em Desenvolvimento Regional) - Universidade Tecnológica Federal do Paraná, Pato Branco, PR, 2017.

COMISSÃO DE CIÊNCIA, TECNOLOGIA, INOVAÇÃO, COMUNICAÇÃO E INFORMÁTICA - CCT. Relatório de avaliação de políticas públicas: fundos de incentivo ao desenvolvimento científico e tecnológico. Brasília, DF, 2016.

COMISSÃO DE CIÊNCIA, TECNOLOGIA, INOVAÇÃO, COMUNICAÇÃO E INFORMÁTICA - CCT. Relatório de avaliação de políticas públicas: Programa Ciência sem Fronteiras. Brasília, DF, 2015. 
CRUZ, V. X. A. Programa Ciência sem Fronteiras: uma avaliação da política pública de internacionalização do ensino superior sob a perspectiva do paradigma multidimensional. 2016. Dissertação (Mestrado em Administração Pública) - Faculdade de Ciências e Tecnologia, Universidade Federal de Goiás, Goiânia, GO, 2016.

CUNHA, D. A. Ciência sem Fronteiras: perspectivas da internacionalização e a experiência australiana. 2016. Tese (Doutorado em Educação em Ciências) - Instituto de Ciências Básicas da Saúde, Universidade Federal do Rio Grande do Sul, Porto Alegre, RS, 2016.

DE WIT, H. et al. Internationalisation of higher education. Brussels: European Parliamant's Committee on Culture and Education, European Union, 2015.

ENGBERG, D. et al. The rationale for sponsoring students to undertake international study: an assessment of national student mobility scholarship programmes. London: British Council, 2014.

FAGUNDES, C.; LUCE, M. B.; SILVEIRA, P. D. A qualidade da mobilidade de estudantes de Graduação no "Ciências sem Fronteiras". Ensaio: Avaliação e Políticas Públicas em Educação, Rio de Janeiro, v. 27, n.105, p. 904-927, out.-dez. 2019. https://doi.org/10.1590/s0104-40362019002701446

FERNÁNDEZ, E. Política educacional: percepções dos estudantes da área de Engenharia Elétrica e dos Coordenadores do Programa Ciência sem Fronteiras. 2016. Tese (Doutorado em Educação em Ciências) - Universidade Federal do Rio Grande do Sul, Porto Alegre, RS, 2016.

FREY, K. Políticas públicas: um debate conceitual e reflexões referentes à prática da análise de políticas públicas no Brasil. Planejamento e Políticas Públicas, Brasília, DF, n. 21, p. 211-259, jun. 2000. Disponível em: http://www.ipea.gov.br/ppp/index.php/PPP/article/view/89/158. Acesso em: 3 jun. 2018.

GRANJA, C. D. Internacionalização e mobilidade estudantil: o programa Ciência sem Fronteiras na Universidade Estadual de Campinas. 2018. Dissertação (Mestrado em Política Científica e Tecnológica) - Universidade Estadual de Campinas, Campinas, 2018. 
GRIECO, J. A. Fostering cross-border learning and engagement through study abroad scholarships: lessons from Brazil's Science without Borders program. 2015. Dissertação (Master of Arts) - University of Toronto, Toronto, 2015.

GURUZ, K. Higher education and international student mobility in the global knowledge economy. Albany, Suny, 2008.

JANNUZZI, P. Monitoramento analítico como ferramenta para aprimoramento da gestão de programas sociais. Revista Brasileira de Monitoramento e Avaliação, Brasília, DF, n. 1, p. 36-55, jan.-jun. 2011. Disponível em: https://issuu.com/sagi_mds/docs/rbma_01. Acesso em: 3 jun. 2018.

KNIGHT, J. An internationalization model: responding to new realities and challenges. In: WIT, H. et al. (eds.). Higher education in Latin America: the international dimension. Washington, DC: World Bank, 2005. p. 1-38.

KNIGHT, J. Internationalization remodeled: definition, approaches, and rationales. Journal of Studies in International Education, v. 8, n. 1, p. 5-31, Mar. 2004. https://doi.org/10.1177/1028315303260832

KNOBEL, M. Brazil seeks academic boost by sending students abroad. In: MIHUT, G., ALTBACH, P. G., WIT, H. (Eds.). Understanding higher education internationalization: global perspectives on higher education. Rotterdam: Sense, 2012. p. 147-149. (International higher education, v. 66).

KOELLER, P.; VIOTTI, R. B.; RAUEN, A. Dispêndios do Governo Federal em C\&T e P\&D: esforços e perspectivas recentes. Radar, Brasília, DF, n. 48, p. 13-18, dez. 2016. Disponível em: http://www.ipea.gov.br/portal/images/ stories/PDFs/radar/20170110_radar_48_art2.pdf. Acesso em: 21 mar. 2017.

LAGE, T. S. R. Políticas de internacionalização da Educação Superior na região norte do Brasil: uma análise do programa Ciência sem Fronteiras na Universidade Federal do Tocantins. 2015. Dissertação (Mestrado em Desenvolvimento Regional) - Universidade Federal do Tocantins, Palmas, 2015.

LIMA, D. C. C. Olhares sobre o Programa Ciência sem Fronteiras: uma contribuição à gestão pública do fomento à ciência, tecnologia e inovação. 2016. Tese (Doutorado em Educação em Ciências) - Instituto de Ciências Básicas da Saúde, Universidade Federal do Rio Grande do Sul, Porto Alegre, 2016. 
LOPES, B.; AMARAL, J. N.; CALDAS, R. W. Políticas públicas conceitos e práticas. Belo Horizonte: Sebrae/MG, 2008.

MANÇOS, G. D. R. Mobilidade acadêmica internacional e colaboração cientifica: subsídios para avaliação do programa Ciência sem Fronteiras. 2017. Dissertação (Mestrado em Ciências) - Escola de Artes, Ciências e Humanidades, Universidade de São Paulo, São Paulo, 2017.

MARQUES, F. Experiência encerrada: o programa de intercâmbio Ciência sem Fronteiras, que gastou R \$ 13,2 bilhões, a maior parte com bolsas de Graduação no exterior, deixa de existir. Revista Fapesp, São Paulo, n. 256, p. 2-9, jun. 2017.

\section{MILHOMEM, R. B. B. Implementação do Programa Ciência sem} Fronteiras no Estado do Tocantins: limites, desafios e potencialidades. 2016. Dissertação (Mestrado em Gestão de Políticas Públicas) - Universidade Federal do Tocantins, Palmas, 2016.

PEREIRA, V. M. Relatos de uma política: uma análise sobre o Programa Ciência sem Fronteiras. 2013. Dissertação (Mestrado em Desenvolvimento Sustentável) - Centro de Desenvolvimento Sustentável, Universidade de Brasília, Brasília, DF, 2013.

RAMOS, A. G. Programa Ciência sem Fronteiras: desdobramentos e análise das possíveis contribuições à formação acadêmica dos bolsistas. 2016. Dissertação (Mestrado em Educação) - Pontifícia Universidade Católica de São Paulo, São Paulo, 2016.

RESCHK, M. J. D.; BIDO, M. C. F. Potencializando a experiência de internacionalização: reflexões sobre o Programa Ciência sem Fronteiras. Espaço Pedagógico, Passo Fundo, v. 24, n. 1, maio 2017. https://doi.org/10.5335/rep.v24i1.6997

SAENGER, E. C. A bolsa pesquisador visitante especial no Programa Ciência sem Fronteiras no CNPq e a Internacionalização da Ciência. 2016. Dissertação (Mestrado em Educação em Ciências) - Instituto de Ciências Básicas da Saúde, Universidade Federal do Rio Grande do Sul, Porto Alegre, 2016.

SAENGER, E. C.; TEIXEIRA, M. R. F. A internacionalização por meio da bolsa de Pesquisador Visitante Especial do Programa Ciência sem Fronteiras do CNPq. Ensaio: Avaliação e Políticas Públicas em Educação, Rio de Janeiro, v. 26, n. 100, p. 849-868, jul/set. 2018. https://doi.org/10.1590/s0104-40362018002601103 
SECCHI, L. Ciclo de políticas públicas. In: SECCHI, L. Políticas públicas: conceitos, esquemas de análise, casos práticos. São Paulo, SP: Cencage Learning, 2012. p. 33-60.

SOUZA, C. Políticas públicas: uma revisão da literatura. Sociologias, Porto Alegre, n. 16, p. 20-45, jul.-dez. 2006. https://doi.org/10.1590/S1517-45222006000200003

STALLIVIERI, L. Brazil's science without borders program. The Word View, 31 maio 2015. Disponível em: https://www.insidehighered.com/blogs/worldview/brazils-science-without-borders-program. Acesso em: 17 jan. 2017.

TONELLI, E.; WINGLER, S. S.; UEBE MANSUR, A. F. Regressos do Programa Ciência sem Fronteiras: impactos da internacionalização da Educação Superior no Brasil. In: CONGRESSO LATINOAMERICANO DE HUMANIDADES, 12., 2016, Campos dos Goytacazes, RJ, Brasil. Anais[...] v. 1, n. 1, p. 1089-1108, 2016.

UNESCO INSTITUTE FOR STATISTICS - UIS. International student mobility in tertiary education. Disponível em: http://data.uis.unesco.org/. Acesso em: 3 nov. 2016.

U.S. DEPARTMENT OF STATE - USDOS. 100,000 Strong in the Americas. 2013. Disponível em: https://www.state.gov/p/wha/rt/100k/. Acesso em: 23 jun. 2018.

VELHO, L.; RAMOS, M. Y. Internacionalização da ciência no Brasil e mobilidade internacional: políticas, práticas e impacto. In: MARINHO, M.G G. S. M. C. et al. Abordagens em ciência, tecnologia e sociedade. Santo André: Universidade do ABC, 2014. p. 263-287.

VERBIK, L.; LASANOWSKI, V. International student mobility: patterns and trends former deputy director. Bulletin National Higher Education Research Institute, v. 44, p. 8-10, 2007. Disponível em: https://core.ac.uk/download/ pdf/83543455.pdf. Acesso em: 11 fev. 2016.

VIANA, A. L. Abordagens metodológicas em políticas públicas. Revista de Administração Pública, Rio de Janerio, v. 30, n. 2, p. 5-43, mar.-abr. 1996. Disponível em: http://bibliotecadigital.fgv.br/ojs/index.php/rap/article/ view/8095/6917. Acesso em: 29 maio 2018. 


\section{Informações sobre as autoras}

Cintia Denise Granja: Doutoranda no programa em Inovação, Economia e Governança na United Nations University - Maastricht Economic and Social Research Institute on Innovation and Technology. Contato: granja@merit.unu.edu

iD https://orcid.org/0000-0001-5791-1305

Ana Maria Carneiro: Doutora em Política Científica e Tecnológica pela Universidade Estadual de Campinas. Pesquisadora no Núcleo de Estudos de Políticas Públicas e Professora Participante do Programa de Pós-Graduação em Política Científica e Tecnológica da mesma universidade. Contato: anamacs@unicamp.br

(iD) https://orcid.org/0000-0001-6688-1881 